medication may be warranted in patients who also have unprovoked seizures. The authors suggest that VGRS are more frequent than commonly recognized.

\title{
PERISYLVIAN POLYMICROGYRIA EPILEPTIC SYNDROME
}

The epileptic spectrum and EEG findings in 31 patients with a congenital bilateral perisylvian syndrome are reported from the University of Alabama at Birmingham and the CBPS Multicenter Collaborative Study Group. The syndrome was characterized by pseudobulbar palsy, cognitive deficits, polymicrogyria and seizures. Associated malformations (eg. arthrogryposis, club feet) were present in $30 \%$ patients. Seizures present in 27 (87\%) began between 1 month and 14 years of age (mean, 7.9 years). Seizure patterns were varied and mainly consistent with secondary generalized epilepsy; infantile spasms occurred in 4, and partial seizures in 7 (26\%). EEG abnormalities were generalized spike and wave and multifocal discharges in 7 , and multifocal patterns in $10(39 \%)$. CT and MRI showed symmetric bilateral perisylvian cortical thickening. Seizures were unresponsive to AEDs in $65 \%$. Callosotomy in 7 with intractable epilepsy and drop attacks was partially effective; drop attacks ceased in 4. (Kuzniecky $\mathrm{R}$ et al. The epileptic spectrum in the congenital bilateral perisylvian syndrome. Neurology March 1994;44:379-385). (Reprints: Dr Ruben Kuzniecky, Department of Neurology, University of Alabama at Birmingham, UAB Station, Birmingham, AL 35294).

COMMENT. The authors and study group report a developmental syndrome characterized by congenital pseudobulbar palsy, epilepsy, mental retardation, and perisylvian polymicrogyria. Patients with drop attacks may respond to callosotomy when antiepileptic drugs are ineffective.

\section{SOMATOSENSORY EVOKED SPIKES AND EPILEPSY}

The relation between EEG paroxysms evoked by tapping of feet or hands (ES), seizures and epileptic syndromes in 186 children is reported from the Department of Neuropsychiatry, Pontificia Universidade Catolica de Campinas, Brazil. Febrile convulsions alone occurred in 31 (17\%) and nonfebrile seizures in $44(24 \%) ; 111$ were without seizures. The incidence of epileptiform activity in the EEG among these 3 groups with somatosensory evoked spikes (ES) was as follows: $89 \%$ for those with epilepsy, $81 \%$ for children with febrile convulsions, and $40 \%$ for the nonepileptic group. Nonfebrile convulsions occurred in 24 (19\%) of 127 patients with ES compared to only $12(9 \%)$ in a control group with normal EEG. Epileptic syndromes associated with ES included benign childhood epilepsy with centrotemporal spikes in $12(27 \%)$, localization related symptomatic in $4(9 \%)$, and cryptogenic in $22(50 \%)$. (Fonseca LC, Tedrus GMA. Epileptic syndromes in children with somatosensory evoked spikes. Clin Electroencephalogr April 1994;25:54-58). (Reprints: Lineu C Fonseca MD, Rua Sebastiao de Souza 205, cj. 122, CEP-13.020.020, Campinas-Sao Paulo, Brazil).

COMMENT. These authors have previously reported an association between febrile convulsions and somatosensory evoked spikes, mainly in children with epileptiform activity in the EEG. The present study confirms this finding for nonfebrile convulsions by comparing patients with ES and a control group with normal EEG. Children with 
complicated initial febrile seizures and ES are also at greater risk of developing epilepsy

\section{EPILEPSY AND COGNITIVE PERFORMANCE}

Changes in left temporal interictal epileptiform activity during and after the performance of cognitive tasks are reported in an 18-year-old male with intractable complex partial seizures evaluated at the Radcliffe Infirmary, Oxford, UK. Epileptiform discharges were suppressed or enhanced depending on the nature and timing of the tasks. A posterior temporal spike focus occurred only during rest periods that followed verbal tasks. The mean left mid-to-anterior temporal spike count was halved during verbal tasks compared to the count during visuo-spatial tasks. A relatively low rate of right-sided temporal discharges was unchanged during or after the performance of tasks. (Boniface SJ et al. Changes in focal interictal epileptiform activity during and after the performance of verbal and visuospatial tasks in a patient with intractable partial seizures. I Neurol Neurosurg Psychiatry Feb 1994; $57: 227-228$ ). (Respond: Dr SJ Boniface, Dept of Clinical Neurophysiology, Radcliffe Infirmary, Woodstock Road, Oxford OX2 6HE, UK).

COMMENT. Activation or suppression of EEG focal spike discharges in relation to psychological testing may have a role in the medical treatment of certain children with reading and other learning disabilities as well as the assessment of patients before and after surgery for epilepsy.

\section{ANTIEPILEPTIC DRUGS}

\section{PHENYTOIN AND COGNITIVE-MOTOR PERFORMANCE}

Cognitive-motor function in 51 children with seizures well controlled with phenytoin (PHT) monotherapy was assessed in relation to drug concentration, seizure type, and time of medication at the Departments of Psychiatry and Pharmacology, Auckland University School of Medicine, Australia. Age ranged from 4 to 14 years, and performance was significantly better in older patients. Diagnosis (partial vs generalized epilepsy), PHT concentration levels, and change from trough to peak concentration days had little effect. Fluctuations in PHT as great as $50 \%$ had no or minimal effects on performance of tests in low therapeutic doses. (Aman MG, Werry JS et al. Effects of phenytoin on cognitive-motor performance in children as a function of drug concentration, seizure type, and time of medication. Epilepsia Jan/Feb 1994;35:172-180). (Reprints: Dr MG Aman, Nisonger Center, Ohio State University, 1581 Dodd Dr, Columbus, OH 43210).

COMMENT. Maintenance phenytoin monotherapy, at relatively low therapeutic levels, had negligible or no effects on cognitive motor function in a group of children with well controlled seizures.

Performance swings resulting from drug absorption and elimination were absent or minimal in this carefully monitored study. The importance of frequent determinations of phenytoin levels during evaluations of neuropsychological function in children is evident from the following report. 